\title{
Propagation of Fatigue Cracks in Aluminium at Low Temperatures
}

\author{
By Tsugio Ogura*, Seiichi Karashima**, and Kuniyasu Tsurukame***
}

\begin{abstract}
Propagation behaviour of fatigue cracks in aluminium was examined at room temperature, $201^{\circ} \mathrm{K}$, and $78^{\circ} \mathrm{K}$. The purpose of the present investigation is to make clear the fatigue mechanism and also to confirm whether or not the vacancy absorption model for the growth of fatigue cracks, which has been proposed by the present authors, is valid in the case of low temperature fatigue as well.

Slip-line-concentrating region around a fatigue crack was found to be considerably smaller at $201^{\circ} \mathrm{K}$ and $78^{\circ} \mathrm{K}$ than at room temperature. It was also clarified that a power law, $d l / d N=C_{1} K_{I}^{n}$, well-known at room temperature, also holds between the rate of fatigue crack growth, $d l / d N$, and the stress intensity factor, $K_{I}$. In the temperature range examined the growth rate was remarkably affected by temperature, which is expressed by an Arrhenius type equation, $d l / d N=C_{2} K_{I}^{n} \exp (-Q / k T)$ where $Q=0.03 \sim 0.04 \mathrm{eV}$.

These results suggest that it is important to take into account a thermally activated process in considering the mechanism of fatigue crack growth. In conclusion, it is shown that the strong temperature dependence of the growth rate can satisfactorily be explained by the vacancy absorption model, provided that the diffusion of vacancies along dislocation cores is assumed.
\end{abstract}

(Received May 17, 1974)

\section{Introduction}

Various kinds of mathematical equations have been proposed to formulate the fatigue life and propagation rate of fatigue cracks ${ }^{(1)}$. Many of them have taken into account the elastic-plastic behaviour of crack tip material. One of the significant disadvantages of these theories seems to lie in the fact that experimental results on dislocation structure around fatigue cracks have scarecely been considered.

On the basis of the results obtained by microstructural observations with an X-ray microbeam technique ${ }^{(2)}$ and transmission electron microscopy ${ }^{(3)(4)}$, a vacancy absorption model has recently been proposed by the present authors for the growth of fatigue cracks in aluminium ${ }^{(5)(6)}$. In this model it was suggested that the growth of a fatigue crack is

* The Research Institute for Iron, Steel and Other Metals, Tohoku University, Sendai 980, Japan.

** Department of Materials Science, Faculty of Engineering, Tohoku University, Sendai 980 , Japan.

*** Graduate School, Tohoku University, Sendai. Present adress: Central Engineering Laboratories, Nissan Motor Co., Ltd., Yokosuka 237, Japan.

Trans. JIM caused by the absorption of vacancies produced near the fatigue crack during stress cycling and that the vacancies are forced to migrate towards the tip of the fatigue crack with the aid of the driving force due to the stress gradient there. According to this model it is expected that the growth rate of fatigue cracks is strongly affected by testing temperature, because the vacancy migration is the rate-controlling process of the growth.

The effects of temperature on fatigue crack growth were examined for the first time by $\mathrm{Wei}^{(7)}$ in a high strength aluminium alloy. His work, however, has been carried out only over a narrow temperature range $\left(295 \sim 380^{\circ} \mathrm{K}\right)$. The present study aims at obtaining a knowledge of propagation behaviour of fatigue cracks in a much wider and lower range of temperatures to confirm the validity of the vacancy absorption model for fatigue crack propagation.

\section{Experimental Procedure}

The material used was polycrystalline aluminium of $99.99 \%$ purity induction-melted in air. Fatigue specimens shown in Fig. 1 were prepared from $2 \mathrm{~mm}$ cold-rolled strips of the material. The specimens fully annealed and then

1975 Vol. 16 
electropolished were subjected to push-pull fatigue with zero mean stress. The average grain size after the annealing was about $40 \mu$ in diameter. Cyclic frequency was 1800 cycles/ min. Fatigue stresses and testing temperatures are summarized in Table 1. Crack length was measured by using an optical microscope attached to the fatigue machine as shown in Photo. 1. Before the fatigue test a notch was made chemically on the side surface of the specimen to fix the initiation site of a fatigue crack in order that successive measurements of crack length are permitted. The depth and root radius of the notch were $0.5 \mathrm{~mm}$ and $0.1 \mathrm{~mm}$, respectively.

\section{Experimental Results}

The formation of slip bands near a fatigue crack is shown in Photo. 2 for a specimen fatigued at $78^{\circ} \mathrm{K}$ under a stress amplitude of $\pm 4.2 \mathrm{~kg} / \mathrm{mm}^{2}$. As seen in the photograph, dense fatigue slip bands are formed within a narrow region around the crack. A closer examination of fatigue cracks showed that surface crack propagates frequently along the slip bands. The formation of such slip-lineconcentrating region around a fatigue crack and the growth of surface fatigue cracks along slip bands have also been found in a specimen fatigued at room temperature. However, there exist some differences in the propagation behaviour between fatigues at room temperature and at low temperatures. Slip bands around

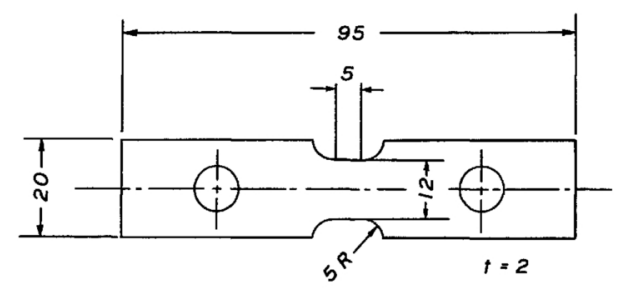

Fig. 1 Fatigue specimen (dimension in $\mathrm{mm}$ ).

Table 1 Conditions of fatigue tests.

\begin{tabular}{ccccccc}
\hline Test. temperature $\left({ }^{\circ} \mathrm{K}\right)$ & \multicolumn{6}{c}{ Fatigue stress, $\sigma_{f}(\mathrm{~kg} / \mathrm{mm})^{2}$} \\
\hline R. T. $(\sim 300)$ & 1.5 & 1.9 & 2.1 & & & \\
201 & 1.5 & 2.1 & 2.3 & 2.5 & 2.7 & 3.2 \\
78 & 4.2 & 5.3 & 6.3 & 6.5 & 6.7 & 7.1 \\
\hline
\end{tabular}

a fatigue crack formed at room temperature were more dense and distributed in a wider region compared with those formed at low temperatures; the size of the region formed at $78^{\circ} \mathrm{K}$ was only about $0.05 \mathrm{~mm}$ as seen in Photo. 2 , while it was reported to be about $1 \mathrm{~mm}$ at room temperature ${ }^{(2)}$. These results suggest that in aluminium the mechanism of fatigue crack propagation at low temperatures is probably the same as that at room temperature, but that the energy dissipation during the crack growth is extremely small at low temperatures.

Examples of plots of crack length, $l$, on a logarithmic scale against the number of stress cycle, $N$, are shown in Figs. 2 and 3 for fatigue tests at $201^{\circ} \mathrm{K}$ and $78^{\circ} \mathrm{K}$, respectively. Such a crack propagation curve for fatigue tests at room temperature has already been reported

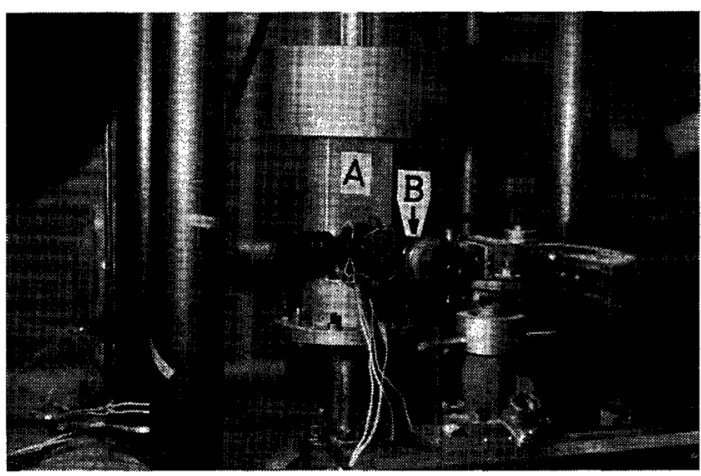

Photo. 1 The apparatus for low temperature fatigue tests and measurement of crack length. (A: low temperature cell containing a fatigue specimen, B: optical microscope to measure crack length through a window of the low temperature cell).

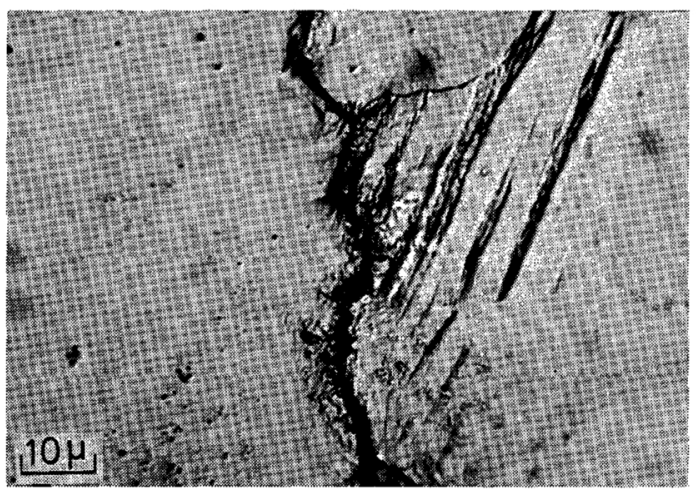

Photo. 2 Slip-line-concentrating region near a fatigue crack in a specimen fatigued at $78^{\circ} \mathrm{K}$. 


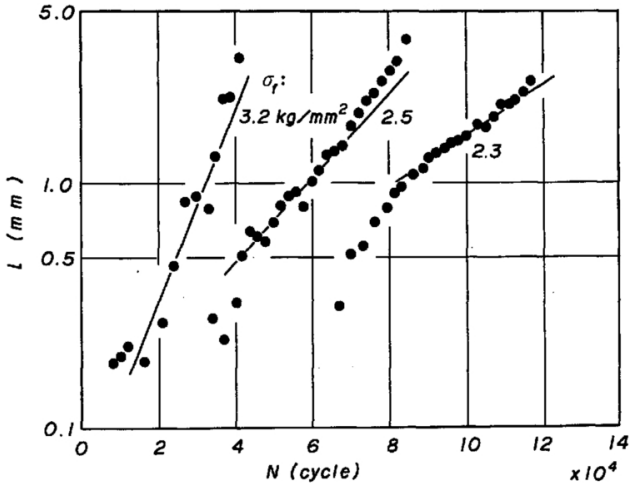

Fig. 2 Examples of fatigue crack propagation curves for fatigue tests at $201^{\circ} \mathrm{K}$.

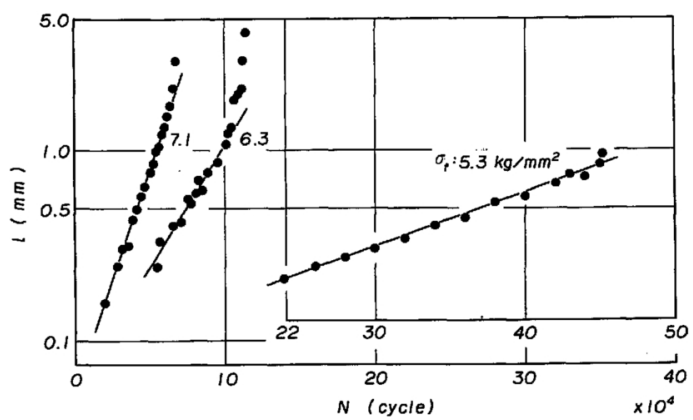

Fig. 3 Examples of fatigue crack propagation curves for fatigue tests at $78^{\circ} \mathrm{K}$.

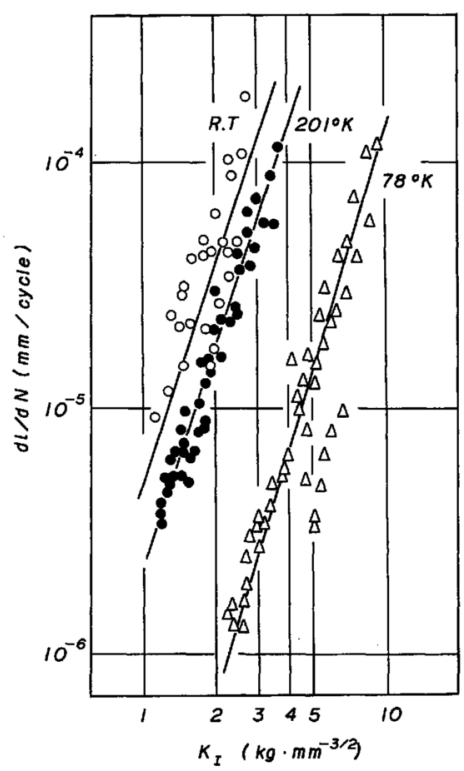

Fig. 4 Rate of fatigue crack propagation at three different temperatures as a function of stress intensity factor. on the same material ${ }^{(2)}$. As in the test at room temperature, linear relations between $\log l$ and $N$ were seen to be realized at low temperatures after a certain number of stress cycles.

The rate of fatigue crack propagation, $d l / d N$, as a function of stress intensity factor, $K_{I}$, is shown in Fig. 4, where $K_{I}$ is calculated using a simplified equation, $K_{I}=\sigma_{f} \sqrt{1}\left(\sigma_{f}\right.$ : the fatigue stress amplitude). The relationships between $d l / d N$ and $K_{I}$ at the three testing temperatures can be expressed by the following equation:

$$
d l / d N=C_{1} K_{I}^{n}, \quad n \cong 3.3
$$

where $C_{1}$ is a constant. It is easily seen in this figure that the propagation rate of fatigue crack is strongly affected by the testing temperature used; for the same $K_{I}$ the propagation rate at room temperature is about $10^{2}$ times as large as that at $78^{\circ} \mathrm{K}$. It is also noteworthy that the strong effect of temperature on $d l / d N$ is largely due to the change in the constant $C_{1}$ with testing temperature and that the exponent $n$ is not affected by changes of temperature. These results are in good agreement with that recently obtained on polycrystalline copper ${ }^{(8)}$.

The power relation between $d l / d N$ and $K_{I}$ shown in Fig. 4 has generally been known for fatigue tests at room temperature on various materials $^{(9)}$. This fact supports the aforementioned suggestion that the mechanism of fatigue crack propagation of aluminium at lower temperatures is substantially the same as that at room temperature. It may be permitted, therefore, to discuss the propagation of aluminium fatigue cracks at low temperatures from the same standpoint as that at room temperature.

\section{Discussion}

The remarkable effect of temperature on the propagation rate of fatigue cracks, which was demonstrated in the present experiments, tacitly indicates that the propagation of fatigue cracks is controlled by a thermally activated process. In order to ascertain this indication the measured rate of fatigue crack propagation is replotted in Fig. 5 on a logarithmic scale against the inverse of testing temperature. Although the scatters of the values of $d l / d N$ were shown by the vertical bars only for the 


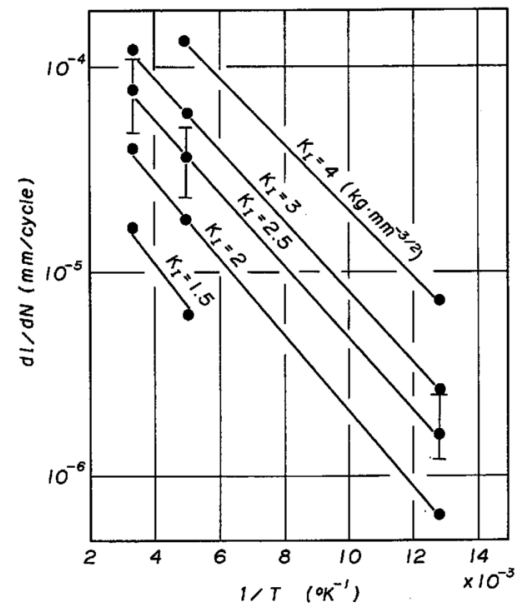

Fig. 5 Relationship between propagation rate of fatigue crack and testing temperature.

case of $K_{I}=2.5 \mathrm{~kg} / \mathrm{mm}^{3 / 2}$, scatters for the other $K_{I}$ 's were nearly the same as them. As seen in the figure, temperature dependence of the growth rate of fatigue crack is given by an Arrhenius type equation:

$$
d l / d N=C_{2} K_{I}^{n} \exp (-Q / k T),
$$

where $C_{2}$ is a constant and $Q$ the apparent activation energy. The value of $Q$ seems to increase slightly with decreasing stress intensity factor, ranging from 0.03 to $0.04 \mathrm{eV}$ in the present measurements. These $Q$-values are covered by those obtained in Wei's experiments $^{(7)}$, where the dependence of $Q$ on $K_{I}$ was more remarkable. It is concluded from these experimental results that the propagation of fatigue cracks is controlled by a thermally activated process.

Assuming a thermally activated process, several theoretical analyses have recently been made to derive a mathematical formulation for the growth rate of fatigue $\operatorname{crack}^{(5)(10)}$. For example, the growth rate due to the vacancy absorption model ${ }^{(5)}$ is given by

$$
d l / d N=C_{3} K_{I}^{m} T^{-2} \exp \left(-2 E_{m} / k T\right)
$$

$\dagger$ Taking account of temeprature dependence of various parameters contained in the constant $C_{3}$, eq. (3) can be written more accurately: $\mathrm{d} l / \mathrm{d} N=C_{3}^{\prime} K_{I}^{m} T^{p-2}$ exp $\left(-2 E_{m} / k T\right)$. In the following discussion, however, $p$, probably being very small, is assumed to be zero, since the accurate estimation of its value is difficult at present. where $C_{3}$ is a constant depending on both materials and experimental conditions, $m$ the exponent depending on cyclic strain hardening exponent $^{(11)}$ of the material, and $E_{m}$ the activation energy for migration of vacancies.

Another type of theoretical analysis has been made on the basis of the models taking into account the elastic-plastic behaviour of material around crack tip ${ }^{(9)(12) \sim(15)}$. In the theory of Weertman ${ }^{(14)}$, for example, the growth rate of fatigue crack is given by

$$
d l / d N=C_{4} l^{2} \sigma_{f}^{4} / \gamma \mu \sigma_{i}^{2}
$$

where $C_{4}$ is a constant, $\sigma_{f}$ the fatigue stress amplitude, $\gamma$ the energy stored at the tip of a crack before its growth, $\mu$ the shear modulus, and $\sigma_{i}$ the frictional stress against dislocation sliding within a plastic zone. Using the equation $K_{I}=\sigma_{f} \sqrt{l}$ and assuming that the temperature dependence of the term, $\gamma \mu$ is very weak, eq. (4) is rewritten as

$$
d l / d N=C_{5} K_{I}^{4} / \sigma_{i}^{2}
$$

Therefore, the effect of temperature on $d l / d N$ in Weertman's theory should be judged from those on $\sigma_{i}^{\dagger \dagger}$.

It has been shown that the growth rate of fatigue crack at room temperature can be explained fairly well by the vacancy absorption model if vacancy diffusion along dislocation cores is assumed ${ }^{(5)}$. It has been reported that the theory of Weertman ${ }^{(14)}$ is also useful for a quantitative explanation of the growth rate of fatigue crack at room temperature. It is interesting to examine whether or not they can also explain the growth rate of fatigue crack at low temperatures. Determining the constants $C_{3} K_{I}^{m}$ in eq. (3) and $C_{5} K_{I}^{4}$ in eq. (5) so that the theoretical values fit the experimental ones obtained at room temperature, the growth rates at low temperatures were calculated. The results are

i† According to the present observations on slipline-concentrating regions around fatigue cracks, $\gamma$ in eq. (4) may greatly decrease with decreasing temperature, as already suggested. If this is the case, the effect of temperature due to the change of $\sigma_{i}$ may be greatly weakened by the counter-effect due to the change of $\gamma$. 


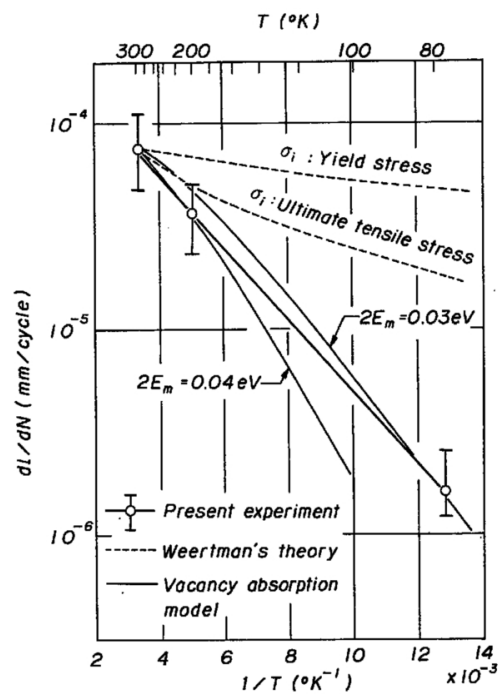

Fig. 6 Comparison of measured growth rates of fatigue crack obtained in the present experiment with those predicted by Weertman's theory or by vacancy absorption model.

shown in Fig. 6†t†. As seen in the figure Weertman's theory is difficult to explain the steep decrease of the growth rate at low temperatures. On the other hand, the rates predicted by the vacancy absorption model coincide well with the experimental data, provided the value of $0.03 \sim 0.04 \mathrm{eV}$, which is obtained in the present experiments, is used for $2 E_{m}$.

The vacancy absorption model can thus explain the temperature dependence of the growth rate of aluminium fatigue crack below room temperature. However, a rough estimation $^{(5)}$ has shown that the energy of vacancy migration is, if pipe diffusion is assumed, about $0.18 \mathrm{eV}$, which is 5 times as large as the apparent activation energy obtained in the present experiment. In considering the reason why the apparent activation energy is so small, it is very suggestive to take into account the fact revealed by $\mathrm{Wei}^{(7)}$ that the energy has a tendency to increase with decreasing stress intensity factor. Although no definite conclusion can be drawn with regard to this problem, it

$\dagger^{+} \dagger$ Yield or ultimate tensile stress was taken for $\sigma_{i}$ in eq. (5). Data on the temperature dependence of the mechanical properties reported by Knöll and Macherauch ${ }^{(14)}$ in aluminium were used in this calculation. seems very probable that the stress concentration at the tip of a fatigue crack lowers the apparent activation energy for vacancy migration in this region.

From the considerations described so far, it is concluded that the vacancy absorption model is very promising for a thermally activated process in fatigue crack growth.

\section{Conclusions}

Propagation behaviour of fatigue cracks in aluminium was examined at room temperature, $201^{\circ} \mathrm{K}$, and $78^{\circ} \mathrm{K}$ to make clear the fatigue mechanism and also to confirm the validity of vacancy absorption model for fatigue crack growth at low temperatures. The results obtained are summarized as follows:

(1) Slip-line-concentrating region around fatigue cracks was found to be considerably smaller at $201^{\circ} \mathrm{K}$ and $78^{\circ} \mathrm{K}$ than at room temperature. It was also clarified that fatigue cracks on the specimen surface propagate along slip bands within the region at low temperatures.

(2) A power law, $d l / d N=C_{1} K_{I}^{n}$, which has been well-known at room temperature, was confirmed to hold also at low temperatures. It was shown that the constant $C_{1}$ is decreased markedly with decreasing testing temperature and that the exponent $n$ is almost independent of the temperature.

(3) The growth rate was strongly affected by fatigue temperature and was expressed for a given $K_{I}$-value by an Arrhenius type equation:

$$
d l / d N=C_{2} K_{I}^{n} \exp (-Q / k T)
$$

The value of $0.03 \sim 0.04 \mathrm{eV}$ was obtained for the apparent activation energy, $Q$.

(4) The strong effect of temperature on the growth rate revealed in the present experiment was shown to be satisfactorily explained by the vacancy absorption model.

On the basis of these experimental results, it was concluded that the vacancy absorption model is very promising for a thermally activated process in fatigue crack growth.

\section{REFERENCES}

(1) For example, B. Tomkins, G. Summer and J. Wareing: Fracture, Chapman and Hall 
Ltd, (1969), p. 712.

(2) T. Ogura and S. Karashima: Trans. JIM, 13 (1972), 428.

(3) T. Ogura and S. Karashima: ibid., 10 (1969), 292.

(4) T. Ogura and S. Karashima: J. Japan Inst. Metals, 34 (1970), 746.

(5) T. Ogura and S. Karashima: ibid., 35 (1971), 291.

(6) T. Ogura and S. Karashima: Trans. JIM, 15 (1974), 324.

(7) R. P. Wei: Intl. J. Frac. Mech., 4 (1968), 159.

(8) H. Ishii and J. Weertman: Met. Trans., 2 (1971), 3441.

(9) For example, F. A. McClintock: Fracture of
Solids, Interscience Pub., (1963), p. 65.

(10) T. Yokobori: J. Phys. Soc. Japan, 10 (1955), 368.

(11) T. Yokobori and M. Ichikawa: Rep. Res. Inst. Strength and Fracture of Materials, Tohoku Univ., Japan, 4 (1968), 45.

(12) A. K. Head: Phil. Mag., 44 (1953), 925.

(13) P. C. Paris and F. Erdogan: Trans. ASME, D85 (1963), 528.

(14) J. Weertman: Intl. J. Frac. Mech., 2 (1966), 460.

(15) N. E. Frost and J. R. Dixon: ibid., 3 (1967), 301.

(16) H. Knöll and E. Macherauch: Z. Metallk., 60 (1969), 399. 